\title{
MODE OF REFERENCE
}

\section{Manuscripts}

All manuscript references are to documents in the Special Collections of Aberdeen University. By far most of the manuscripts used here are in the Birkwood Collection. In such references, the first four digits, 2131, have been dropped, so that $2131 / 8 / \mathrm{III} / 3$ becomes $8 / \mathrm{III} / 3$, followed, where relevant, by the number of the manuscript page(s) referred to. Other manuscript numbers are given in full.

\section{Published works}

Works are generally cited with shortened titles, while full bibliographical details are given in the Bibliography. References to Hume's Treatise cite Book, Part, Section, Paragraph according to the edition by D. F. and M. J. Norton, followed by the page number(s) of the edition by L. A. Selby-Bigge and P. H. Nidditch. References to Hume's Enquiries cite Part, Section, Paragraph according to the editions by T. L. Beauchamp, followed by the page number(s) of the edition by L. A. Selby-Bigge and P. H. Nidditch. 